\title{
Macroscopic hematuria as a presentation of gestational trophoblastic neoplasia in an adolescent woman
}

\author{
Irene Ruengkhachorn $^{1^{*}}$, Nisarat Phithakwatchara ${ }^{2}$, Usanee Chatchotikawong $^{3}$ \\ ${ }^{1}$ Gynecologic Oncology Division, Department of Obstetrics and Gynecology, Faculty of Medicine, Siriraj Hospital, Mahidol \\ University, Bangkok, Thailand; *Corresponding Author: irene_siriraj@yahoo.com \\ ${ }^{2}$ Maternal Fetal Medicine Division, Department of Obstetrics and Gynecology, Faculty of Medicine, Siriraj Hospital, Mahidol \\ University, Bangkok, Thailand \\ ${ }^{3}$ Department of Obstetrics and Gynecology, Faculty of Medicine, Siriraj Hospital, Mahidol University, Bangkok, Thailand
}

Received 24 April 2013; revised 27 May 2013; accepted 28 June 2013

Copyright (c) 2013 Irene Ruengkhachorn et al. This is an open access article distributed under the Creative Commons Attribution License, which permits unrestricted use, distribution, and reproduction in any medium, provided the original work is properly cited.

\begin{abstract}
Gestational trophoblastic neoplasia (GTN) is a hemorrhagic malignancy with highly rapid potential metastasis via hematogenous spreading. Renal metastasis is of infrequent occurrence. Among previous case reports of renal metastasis in GTN, this is the youngest woman presenting a large renal lesion concurrent with pulmonary and brain metastases. Disease remission had been achieved by treatment with combined chemotherapy and whole brain radiation, although acute myeloblastic leukemia (AML) developed later.
\end{abstract}

Keywords: Gestational Trophoblastic Neoplasia; Choriocarcinoma; Metastasis; Renal; Hematuria

\section{INTRODUCTION}

Gestational trophoblastic neoplasia (GTN), a very hypervascularized tumor, is the most curable gynecologic malignancy. This results from its high sensitivity to chemotherapeutic agents and radiation. GTN has a variety of histopathologies, including invasive mole, choriocarcinoma, and placental site trophoblastic tumor [1]. A serum human chrorionic gonadotropin (serum hCG), is helpful in clinical diagnosis and follow-up assessment after treatment [1,2]. Abortion is the most common manifestation. Other atypical presenting symptoms are alteration of consciousness or neurological deficit due to brain metastasis, dyspnea or respiratory failure from pulmonary metastasis, etc. A history of previous molar pregnancy is a significant clue, making the diagnosis much easier. On the other hand, women without antecedent gestation often have multiple visits to several hospitals with diagnosis failure before getting an accurate diagnosis. An unintended delay in diagnosis could lead to massive bleeding with disseminated intravascular coagulopathy (DIC) and death eventually.

\section{CASE PRESENTATION}

A 17-year-old woman with 6-week duration of amenorrhea, nausea and headache was initially diagnosed as morning sickness at private hospital with symptomatic and supportive treatments. Three weeks later, she had massive gross hematuria and acute urinary retention. Multiple lesions at right kidney, right ovary and both lungs were identified from emergency ultrasonography and computed tomography (CT) of whole abdomen. She was then referred to our institute, a tertiary care center and medical school hospital. In spite of Foley catheterization, she still had urinary retention with large amount of active gross hematuria and felt more fatigued. All of these brought her to our emergency department before urologist appointment. An important history of spontaneous abortion at 8 weeks' gestation in the past 14 months was revealed. Since then, no menstruation had resumed neither with sexual intercourse nor with contraception. She also complained of abnormal vaginal bleeding which had just developed for 2 weeks. Physical examination on admission revealed a conscious, moderately pale and hyposthenic built young lady with body mass index (BMI) of $15.6 \mathrm{~kg} / \mathrm{m}^{2}$. Her heart rate was 118 beats per minute with normotensive blood pressure, 120/70 mmHg. She had marked dyspnea with respiratory rate of 40 per minute, and diminished breath sounds at right lung. She had no neurological symptoms or deficit. 
Slight tenderness at lower abdomen was expressed and a cystic mass was palpable in her right pelvic region. Pelvic and rectal examinations revealed minimal bloody discharge, congested closed cervix, and suspected right adnexal cyst, approximately $10 \mathrm{~cm}$ in diameter. Transabdominal and transvaginal 2D ultrasonography with Doppler study demonstrated a normal-sized uterus, containing a mixed echogenic mass, $3.5 \times 2.7 \mathrm{~cm}$ in diameter, with irregular outlining and high vascularization. Chest X-ray showed multiple pulmonary nodules in both lungs. Her hematocrit was $26 \%$, consistent with the history of continuing blood loss. Serum hCG was reported as higher than $1,000,000 \mathrm{mIU} / \mathrm{mL}$. We made further investigations for GTN staging and scoring, accompanied with general managements including oxygenation, blood transfusion, continuous bladder irrigation and nutritional supplement. Contrast-enhanced CT brain scan demonstrated a multilocular cystic mass, $6.1 \times 4.6 \times 4.1 \mathrm{~cm}$. in size, at grey-white junction of right high parietal lobe, composed of hypodense portions and some enhancing hyperdense areas. These were compatible with intralesional hemorrhage and perilesional vasogenic oedema. From previous abdominal CT images, right pelvicalyceal system was dilated with an ill-defined, non-enhancing, soft tissue-attenuating area at upper pole of right kidney, $8.5 \times 6.5 \mathrm{~cm}$. in diameter. A large multilocular cystic mass, $10 \times 7 \mathrm{~cm}$. in diameter, was also identified in the right pelvic cavity. This patient has no histopathologic diagnosis.

According to the International Federation of Gynecologic and Obstetrics (FIGO) anatomic staging and prognostic scoring system, her diagnosis was GTN stage IV:16 (pulmonary, renal and brain metastases). After discussion with the patient and her family, the combination of etoposide, methotrexate, actinomycin-D, cyclophosphamide, and vincristine (EMA-CO) chemotherapy and whole brain radiation therapy (WBRT) 3000 cGy for initial treatment was started. Hematuria was dramatically improving and disappeared in 10 days with the ability to remove Foley catheter and no urinary retention anymore. Her serum hCG had been decreased subsequently in each cycle of chemotherapy (Figure 1). After the third cycle, both kidneys appeared normal in whole abdominal CT scan. Her general health was unremarkable. Since the second cycle of chemotherapy, she received granulocyte colony-stimulating factors (G-CSFs) for secondary prophylaxis. Findings in chest $\mathrm{X}$-ray and magnetic resonance imaging of brain returned to normal after receiving 15 cycles of chemotherapy. Unfortunately, she developed pancytopenia 6 months after remission. Acute myeloblastic leukemia (AML), M5 type, was then diagnosed from bone marrow study, which has been treated with combined chemotherapy, idarubicin and cytosine arabinoside.

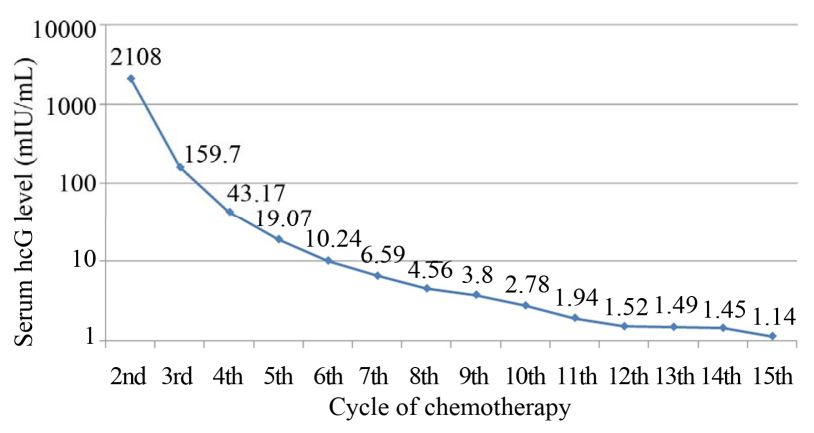

Figure 1. Demonstrated serum hCG level along the course of chemotherapy in this case.

\section{DISCUSSION}

This is a case of GTN with atypical presentations, which had dramatic response to treatment. Most common sites of metastasis in GTN are lungs (80\%), vagina (30\%), liver (10\%), and brain (10\%) [1]. The incidence of renal metastasis ranges from $1 \%$ to $20 \%$, depending on study population, and is high to $10 \%-50 \%$ in autopsy cases $[3,4]$. In one previous study, renal metastasis was found in $1.3 \%$ of patients with GTN and pulmonary metastasis and in $14 \%$ of those referred for salvage chemotherapy [3]. Tissue biopsies of the metastasis sites are not necessary due to hemorrhagic risk [1].

Signs and symptoms of renal involvement could be low back pain, flank pain, gross or microscopic hematuria, and serum hCG-related symptoms such as nausea, vomiting, and fatigue [4-8]. All cases with renal metastasis have concurrent pulmonary metastasis [3-11]. This is hypothesized by the theory of venous embolic invasion and metastasis. Whenever the tumor cells entrap in the lungs, they can spread hematogenously to every part of body as arterial emboli $[12,13]$. Hence, in case of GTN with pulmonary metastasis, metastatic survey for every part of human body should be made, not only for liver and brain metastases.

Several features of renal metastasis demonstrated in CT imaging have been reported, for instance, multiple hypodensity masses [6], a large mixed attenuating soft tissue mass [7], and an enhancing vascular mass [5]. In this case, CT scan demonstrated an ill-defined non-enhancing mass, $8.5 \mathrm{~cm}$ in maximum diameter, which located at upper pole of right kidney. This is a typical characteristic of GTN which has hypervascularity and susceptibility for bleeding. Generally, the appearance of renal metastatic lesion in early stage is a soft tissue mass, which will change if there is either hemorrhage from enormous fragile vessels or necrosis inside [4].

Usually, GTN with renal metastasis was categorized as high-risk, according to the FIGO 2000 modified World Health Organization (WHO) scoring system [310]. Serum hCG is often more than $1,000,000 \mathrm{mIU} / \mathrm{mL}$, 
as in this case $[4,5,9]$. That is why these patients should be treated with etoposide, methotrexate, actinomycin-D, cyclophosphamide, and vincristine (EMACO) regimen. Arterial embolization with concurrent combined chemotherapy was reported as another treatment option with favourable outcomes $[5,6]$. Other coexistent organs should be treated as stated in the standard modality treatment of each organ, for example, systemic combined chemotherapy and intrathecal chemotherapy with whole brain radiation for brain metastasis. Surgical procedure in GTN, resulting in iatrogenic massive hemorrhage and increased morbidity and mortality rate, ought to be avoided. Nevertheless, it may be utilized in an emergency condition, such as bleeding or rupture of lesions.

Prognosis of renal metastasis from GTN is rather fair, depending on concurrent organ metastasis. Based on previous studies, treatment response was reported in 16/21 cases [3-11,14-17]. Post-operative complications and brain or liver metastasis were the causes of death in 2 and 5 cases [7]. The longest remission interval in previous studies was 2 years [8]. In the present case, she has been in remission for 7 months. Therefore, bilateral renal involvement and concurrent liver or brain metastasis are probably the poor prognostic factors in GTN.

Risk of secondary malignancy following high-dose chemotherapy is $1 \%$. Of which, most common malignancies are AML, breast cancer, colon cancer and skin melanoma [18]. A retrospective study in 5510 stage I-III breast cancer patients found that AML or myelodysplastic syndrome (MDS) developed in $1.8 \%$ of patients who received G-CSFs or granulocyte-macrophage colonystimulating factors (GM-CSFs), but only in $0.7 \%$ of patients who did not (hazard ratio $=2.59,95 \% \mathrm{CI}=1.30$ to 5.15) [19]. Furthermore, another systematic review in 2010 by Lyman et al., from 25 randomized controlled trials; reported AML in 43/6058 patients who received G-CSFs and 22/6746 patients who did not (RR 1.92 95\% CI, 1.19 to 3.07) [20]. The present case had rapid development of AML, only 6 months after $2030 \mathrm{mg}$ of etoposide administration. Both etoposide and G-CSFs may synergistically induce AML in this case. Consequently, clinicians should weigh risks against benefits of using these agents together.

\section{CONCLUSION}

Renal metastasis from GTN is uncommon but has favorable outcomes. Clinicians should keep in mind about the possibility of GTN in a reproductive female patient with unexplained bleeding manifestations. Misdiagnosis as renal cell carcinoma would mislead the surgeon to have the operation taken unnecessarily. Doctor recognition and blood test for serum hCG can save patient's life.

\section{ACKNOWLEDGEMENTS}

We appreciate and would like to thank Assist. Prof. Weerasak Wongtiraporn for his contribution to the patient.

\section{REFERENCES}

[1] Berkowitz, R.S. and Goldstein, D.P. (2009) Current management of gestational trophoblastic diseases. Gynecologic Oncology, 112, 654-662. doi:10.1016/j.ygyno.2008.09.005

[2] Soper, J.T. (1995) Identification and management of highrisk gestational trophoblastic disease. Seminars in Oncology, 22, 172-184.

[3] Soper, J.T., Mutch, D.G., Chin, N., Clarke-Pearson, D.L. and Hammond, C.B. (1988) Renal metastases of gestational trophoblastic disease: A report of eight cases. $\mathrm{Ob}$ stetrics and Gynecology, 72, 796-798.

[4] Kutcher, R., Lu, T., Gordon, D.H. and Becker, J.A. (1977) Renal choriocarcinoma metastasis: A vascular lesion. American Journal of Roentgenology, 128, 1046-1048. doi:10.2214/ajr.128.6.1046

[5] Vijay, R.K., Kaduthodil, M.J., Bottomley, J.R. and Abdi, S. (2008) Metastatic gestational trophoblastic tumour presenting as spontaneous subcapsular renal haematoma. British Journal of Radiology, 81, e234-e237. doi:10.1259/bjr/81495647

[6] Yang, D.M., Yoon, M.H., Kim, H.S. and Shin, D.B. (2000) Intrarenal pseudoaneurysms complicating renal choriocarcinoma metastases: Treatment with coil embolization. Clinical Imaging, 24, 217-220. doi:10.1016/S0899-7071(00)00220-5

[7] Chakrabarti, J., Mishra, P.K., Mondal, P., Ghosh, D., Chaterjee, R.G. and Ghosh, D.K.G. (2006) Metastatic choriocarcinoma presenting as a renal mass. Indian Journal of Medical \& Paediatric Oncology, 27, 47-49.

[8] Ghaemmaghami, F. and Ashraf-Ganjoie, T. (2006) Metastatic gestational trophoblastic neoplasms and no gynecological symptoms: Report of five cases. Acta Medica Iranica, 46, 77-80.

[9] Tai, K.S., Chan, F.L. and Ngan, H.Y. (1998) Renal metastasis from choriocarcinoma: MRI appearance. Abdominal Imaging, 23, 536-538. doi:10.1007/s002619900395

[10] Wang, Y. (1991) Clinicopathological study on 31 cases of renal metastasis of choriocarcinoma. Zhonghua Yi Xue Za Zhi, 71, 514-515.

[11] Mack, R.B., Katz, S.M. and Amenta, P.S. (1992) Choriocarcinoma of the kidney. Journal of the American Osteopathic Association, 92, 799-802.

[12] Jones, W.B. and Lewis Jr., J.L., (1974) Treatment of gestational trophoblastic disease. American Journal of $\mathrm{Ob}$ stetrics \& Gynecology, 120, 14-20.

[13] Song, H.Z., Wu, B.Z., Tang, M.Y., Wang, Y.E. and Yang, X.Y. (1984) A staging system of gestational trophoblastic neoplasms based on the development of the disease. Chinese Medical Journal (England), 97, 557-566. 
[14] Mazur, M.T., Lurain, J.R. and Brewer, J.I. (1982) Fatal gestational choriocarcinoma. Clinicopathologic study of patients treated at a trophoblastic disease center. Cancer, 50, 1833-1846.

doi:10.1002/1097-0142(19821101)50:9<1833::AID-CNC $\underline{\text { R2820500930>3.0.CO;2-4 }}$

[15] Lal, A., Singhal, M., Kumar, S., Bag, S., Singh, S.K. and Khandelwal, N. (2009) Bilateral renal and jejunal metastasis of choriocarcinoma presenting as spontaneous renal hemorrhage. Cancer Imaging, 9, 56-58. doi:10.1102/1470-7330.2009.0010

[16] Ogunbiyi, O.A., Enweren, E.O. and Ogunniyi, J.O. (1986) Unusual renal manifestations of choriocarcinoma. African Journal of Medicine and Medical Science, 15, 93-97.

[17] Regis, C., Taieb, S., Lesoin, A., Baranzelli, M.C., Blehaut, T. and Leblanc, E. (2006) Uncommon clinical presentation of a gestational choriocarcinoma. Gynécologie $\mathrm{Ob}$ stétrique \& Fertilité, 34, 716-719. doi:10.1016/j.gyobfe.2006.05.005
[18] Rustin, G.J., Newlands, E.S., Lutz, J.M., Holden, L., Bagshawe, K.D., Hiscox, J.G., et al. (1996) Combination but not single-agent methotrexate chemotherapy for gestational trophoblastic tumors increases the incidence of second tumors. Journal of Clinical Oncology, 14, 27692773.

[19] Hershman, D., Neugut, A.I., Jacobson, J.S., Wang, J., Tsai, W.Y., McBride, R., et al. (2007) Acute myeloid leukemia or myelodysplastic syndrome following use of granulocyte colony-stimulating factors during breast cancer adjuvant chemotherapy. Journal of the National Cancer Institute, 99, 196-205. doi:10.1093/jnci/djk028

[20] Lyman, G.H., Dale, D.C., Wolff, D.A., Culakova, E., Poniewierski, M.S., Kuderer, N.M., et al. (2010) Acute myeloid leukemia or myelodysplastic syndrome in randomized controlled clinical trials of cancer chemotherapy with granulocyte colony-stimulating factor: A systematic review. Journal of Clinical Oncology, 28, 2914-2924. doi:10.1200/JCO.2009.25.8723 